\section{Dermatologie für praktizierende Pädiater}

\author{
J. Küttel, D. Müller, M.-A. Steinemann
}

\begin{abstract}
Selber aktiv geworden
1993 fanden sich frei praktizierende Pädiater zusammen, um praxisnahe Fortbildungen zu organisieren. Anstoss war die Erkenntnis, dass die Klinikausbildung Mängel hat und wir unsere Bedürfnisse selber an die Hand nehmen müssen. Aus diesen Aktivitäten ist das Forum für Praxispädiatrie entstanden. Ein Haupttätigkeitsbereich dieser grossen Gruppierung ist die Organisation praxisbezogener Fortbildungen. Praxisbezogen bezüglich Inhalt, Darbietung und zeitlichem Ablauf. Der Dermatologiekurs, erstmals 1996 durchgeführt, ist eine davon.
\end{abstract}

\section{Wie baut man einen Kurs?}

Die drei als Autoren zeichnenden Kursleiter setzten sich das Ziel, die Dermatologiekenntnisse des Praxispädiaters zu verbessern. Aufgrund persönlicher Bedürfnisse wurde eine Themenliste zusammengestellt. Diese liessen wir an diversen Fortbildungen zirkulieren, um eine breiter abgestützte Gewichtung zu erhalten. Etwa 50 Praktiker setzten so die thematischen Schwerpunkte. Nun suchten wir geeignete Referenten und einen Ort für die Durchführung. Die Vorgabe an die dermatologischen Fachkollegen lautete: Das möchten wir als Kursinhalt; er sollte interaktiv und in Lernschritten vermittelt werden. In Professor Dr. med. Theo Rufli, Chefarzt der Dermatologischen Universitätsklinik Basel, fanden wir einen didaktisch und menschlich begabten Dozenten als fachlichen Kursleiter. Von gleicher Qualität sind auch seine Mitarbeiter. Er machte ohne zu zögern mit und ging bereitwillig auf unsere Wünsche ein. Gemeinsam mit ihm teilten wir die Thematik in drei Blöcke zu zwei Tagen auf und verteilten sie über ein Jahr. Als administrative Stütze für die Kursdurchführung diente uns das Sekretariat des Forums.

\section{Didaktische Merkmale}

Die Teilnehmer sollten möglichst aktiv mitmachen. Deshalb musste die Teilnehmerzahl beschränkt werden. Interaktiver Unterricht war ja eine Kursgrundlage. Man konnte jederzeit Fragen stellen. Nach

Korrespondenz:

Dr. med. Josef Küttel

Ladengasse

CH-6030 Ebikon
20 Minuten war eine Pause fällig, um Ermüdungserscheinungen zu vermeiden. Wir wollten zusätzlich, dass die Kursteilnehmer eigene Fälle aus der Praxis einbringen. Dies geschah in Form einer Diaklinik. Mit Minolta Schweiz konnten wir - ausgelöst durch die Initiative eines engagierten Kursteilnehmers mit Querverbindungen - ein günstiges Set mit Spiegelreflexkamera, Makroobjektiv und Ringblitz anbieten. Es wurden zusammenfassende schriftliche Unterlagen abgegeben. Die Kurse wurden von uns selbst mittels Fragebogen evaluiert. Das ermöglichte von Mal zu Mal eine stetige Verbesserung.

\section{Selbsttragende Kosten}

Die Kurskosten sollten folgendes decken: Zeit- und Materialaufwand der Organisatoren sowie adäquate Honorierung der Dozenten. Für die Übernachtung sollte jeder Teilnehmer selbst aufkommen. Wir entwarfen ein Budget und errechneten so die Kurskosten von etwa Fr. 900.- pro Teilnehmer für total 6 Kurstage. Wir wollten den Kurs ohne Sponsoren finanzieren. Hingegen waren uns diese willkommen als Gastgeber für den gesellschaftlichen Teil und andere Aktivitäten, von denen sie selber auch profitieren konnten. Eine Firma mit starkem Segment in Dermatologie konnte sogar einen fachlichen Beitrag (Galenik) mit hauseigenen Referenten beisteuern.

\section{Es geht weiter}

Der Kurs wurde 1999 zum dritten Mal durchgeführt. Besucht haben ihn total fast 100 praktizierende Pädiater. Bereits bei der Erstausschreibung war er doppelt überbucht, und schon wird eine vierte Auflage gewünscht. Wir stellten übrigens fest, dass andere Organisatoren Inhalte und Struktur "unseres" Kurses kopierten, was für unser Konzept spricht.

In der Evaluation wurde der Kurs von den Teilnehmern als sehr lehrreich und praxisnah taxiert. Die eigene fachliche Kompetenz habe sich deutlich verbessert und mit Freude an dermatologischen Fragestellungen gepaart. Auch die interdisziplinäre Zusammenarbeit und das gegenseitige Verständnis zweier Fachgesellschaften gewannen. Die anfänglichen Befürchtungen von Dermatologen in der Ausbildung, man werde nach den Kursen in der Poliklinik keine Kinder mehr sehen, bestätigten sich nicht. Das Gegenteil trat ein.

Um den Teilnehmern zu ermöglichen «dranzubleiben", werden halbtägige Diakliniken durchgeführt. Die dort gezeigten Bilder stammen alle aus unseren Praxen. Eingeladen wird, wer den Kurs absolviert hat.

Das Forum führt auch andere Kurse für Praxispädiater durch. Nämlich so viele, wie wir Praktiker selbst bereit sind, zu organisieren. Wer etwas auf die Beine stellen möchte, der kann auf die Unterstützung des Vorstandes (ideell) und des Sekretariates (materiell) zählen. Man wende sich an: Sekretariat FPP, Postfach 534, 4512 Bellach, Tel. 03261842 29, Fax 03261807 40, E-mail: forumfpp@sonet.ch. 